\title{
Giant polarized optical properties in type-II ZnTe/CdSe multiple quantum wells induced by interface chemical bonds
}

\author{
Y. F. Chen", W. S. Su, M. H. Ya, and Y. S. Chiu \\ Department of Physics, National Taiwan University, Taipei, Taiwan, Republic of China \\ Received 22 September 2003, revised 25 September 2003, accepted 6 November 2003 \\ Published online 16 February 2004 \\ PACS 72.80.Ey, 78.55.Et
}

Photoluminescence (PL) and photoconductivity (PC) spectra were studied in type-II ZnTe/CdSe multiple quantum wells. It was found that the PL spectrum exhibits a strong in-plane polarization with respect to $\langle 011\rangle$ axis with polarization degree up to $30 \%$. The degree of polarization does not depend on the excitation intensity and temperature, which excludes extrinsic mechanisms related to the observed in-plane anisotropy. The underlying mechanism of the observed polarization dependence of the optical properties was attributed to the inherent property of the orientation of chemical bonds in $\mathrm{ZnTe} / \mathrm{CdSe}$ heterostructures with no common atom. In addition, the PC spectra also display a strong polarization dependence. We therefore point out that polarized PL and PC spectroscopies provide a unique way to probe the effects of the orientation of interface chemical bonds on semiconductor heterostructures.

(C) 2004 WILEY-VCH Verlag GmbH \& Co. KGaA, Weinheim

1 Introduction Anisotropic properties inherently exist in many crystalline solids [1]. Even though they may make it difficult to understand the physical properties of a solid, many interesting phenomena arise from the effects of anisotropy. To have a heterostructure, we need to deposit two different materials together. It therefore breaks the chemical bonds symmetry and creates new anisotriopic properties. Semiconductor heterostructures, in AB/CD-type combination, whose two constituents do not share a common atom, are excellent candidates to search for anisotropic effects. Indeed, the quantum structures without a common atom have nonequivalent normal and inverted interfaces, which can cause in-plane anisotropy not found in a quantum structure with equivalent interface [2]. It is known that semiconductor heterostructures without a common anion or cation often show a type-II band alignment and large band offset in the conduction and valence bands. The indirect transition due to spatially separated electrons and holes therefore is restricted to a very narrow region containing the interface, and the emitted radiation will exhibit the anisotropic characteristic of the interface chemical bonds [3, 4]. In this paper, we report the interface-induced in-plane anisotropy in a new semiconductor heterostructure without common atom, i.e., $\mathrm{ZnTe} / \mathrm{CdSe}$ multiple quantum wells. The degree of polarization in the photoluminescence(PL) spectra as large as $30 \%$ has been observed. Through the study of the temperature and pumping intensity dependences of the polarized PL spectra, we demonstrate that the observed polarization is an intrinsic property of the $\mathrm{ZnTe} / \mathrm{CdSe}$ heterostructure. In addition, we discover that the photoconductivity (PC) spectra are also very sensitive to the polarization of the incident radiation. Unlike the PL spectra, the polarization of the PC spectra can be induced by interface defects, and it covers a wide range of wavelengths. We therefore point out that polarized PC measurement is a very powerful technique to probe anisotropic defects.

* Corresponding author: e-mail: yfchen@phys.ntu.edu.tw, phone: 886-2-33665125, Fax: 886-2-23940752 
2 Experiment All the II-VI heterostructures fabricated until now have been strained-layer system, with the exception of structures involving $\mathrm{HgTe} / \mathrm{CdTe}$ and the more recently studied alloy combinations $\mathrm{ZnCdSe} / \mathrm{ZnMnSe}$ [4]. The discovery that zinc-blende CdSe layers can be grown by molecular beam epitaxy(MBE) prompted several groups to attempt the growth of a new lattice-matched combination, $\mathrm{ZnTe} / \mathrm{CdSe}$ [5]. This system is unique among II-VI heterostructures in that the lattice match is obtained between two binary compounds where neither the anion nor the cation are common. The lattice parameters of ZnTe and cubic CdSe are $6.099 \AA$ and $6.077 \AA$ [5], respectively, leading to lattice mismatch of only $0.3 \%$. Because of the closely matched lattice constants, one expects negligible strain at sharp interface, thus allowing high quality superlattices with ideal interfaces to be grown. This heterostructure thus provides an excellent system for the study of the physics related to interfaces.

The multiple quantum well(MQW) structures were grown by MBE on (100) semi-insulating GaAs substrate after the deposition of about $2.16 \mu \mathrm{m}$ of a ZnTe buffer layer. The MQWs used here contain 20 periods of alternating ZnTe and CdSe layers. The sample consists of a $120 \AA$ ZnTe barrier layer, a $90 \AA$ CdSe well layer, and finally a $900 \AA$ ZnTe cap layer. Details of the growth was described elsewhere [6].

The PL spectra were recorded by a Spectra Pro 300i monochromator, and an InGaAs detector. The sample was placed inside a close-cycle He cryostat. An Ar-ion laser was used as the excitation source. For the PC measurement, ohmic contacts were formed by depositing indium drops to the four corners of the samples, and annealing the sample at $240{ }^{\circ} \mathrm{C}$ for $10 \mathrm{~min}$. A tungsten lamp dispersed by triple-grating monochromator was used as the light source. A constant current was supplied to the sample by a Keithley 236 source measure unit. The conductivity signal was detected as a change in the voltage drop across the sample using a lock-in amplifier. A detailed description of the experimental setup has been given elsewhere $[7,8]$.

3 Results and discussions The energy gap at $10 \mathrm{~K}$ of $\mathrm{ZnTe}$ and $\mathrm{CdSe}$ are $2.4 \mathrm{eV}$ and $1.75 \mathrm{eV}$, respectively [6]. The conduction-band and valence-band offsets are $1.29 \mathrm{eV}$ and $0.64 \mathrm{eV}$, respectively, as determined by X-ray photoelectron spectroscopy [9]. Two types of excitonic transitions are possible in such a type-II heterostructure. One takes place between electrons localized in the CdSe conduction-band wells and holes in the ZnTe wells which is referred as the type-II transition. The other occurs between electron (or hole) subbands confined in the wells and hole (or electron) subbands at above-barrier energies quasiconfined in the barriers. In that case both the electron and the hole states involved in the transition are localized in the same layer which corresponds to the type-I transition. For the spatially indirect transition, it is caused by the wave function overlap between electron and hole across interface. Because the band offset is very large $(1.29 \mathrm{eV})$, the overlap region at interface is as narrow as a few monolayers. Therefore, one should except that the emitted radiation should reflect the anisotropic property of the interface chemical bonds. The interfaces in this quaternary $\mathrm{CdSe} / \mathrm{ZnTe}$ heterostructure consist of $\mathrm{Cd}-\mathrm{Te}$ and $\mathrm{Zn}-\mathrm{Se}$ bonds; either a $(011)$ and a $(0 \overline{1} 1)$ plane. It is obvious that the lower and the upper interfaces of the quantum well are not equivalent with respect to the bond directions. Their contributions to the anisotropy cannot compensate each other [10]. Therefore, the in-plane anisotropy inherently exists in the CdSe/ZnTe MQWs studied here.

Figure 1 shows the PL spectra for the polarization of the emitted radiation along (011) and $(0 \overline{1} 1)$ direction. The peak at $1.03 \mathrm{eV}$ corresponds to the spatially indirect transition denoted as the type-II transition. One of the excellent evidences is that the PL peak energy can be strongly blue-shift with the pumping intensity. As we can see, the PL intensity is very sensitive to the polarization. Figure 2 shows the PL intensity versus the angle of the analyzer. Solid dots are experimental data, and the curve is fit to $\cos ^{2} \theta$. The polarization degree is found to be as large as $30 \%$. This value is too large to be explained by strain or electric field effects [11]. In order to clarify that the observed polarized PL spectra are induced by the anisotropic nature of the interface chemical bonds, we have performed the pumping power and temperature dependences of the polarization. It is found that the degree of polarization is not sensitive to the change of pumping power in the range of $0.1 \mathrm{~W} / \mathrm{cm}^{2}$ to $25 \mathrm{~W} / \mathrm{cm}^{2}$. The degree of polarization is also very stable with respect to the change of temperature from $10 \mathrm{~K}$ to $300 \mathrm{~K}$. These results can be used to rule out extrinsic mechanisms related to the in-plane anisotropy. For example, the built-in electric fields 
caused by unintentional doping will be screened under light irradiation. We can also exclude a significant role of localized states and nonradiative channels in the formation of the in-plane anisotropy, since they will be gradually saturated by the pumping source. We thus conclude that the polarization of the spatially indirect PL in CdSe/ZnTe is an inherent nature of the interface chemical bonds. Polarized PL measurements therefore provide a simple tool to probe interface anisotropy in quaternary heterostructures.

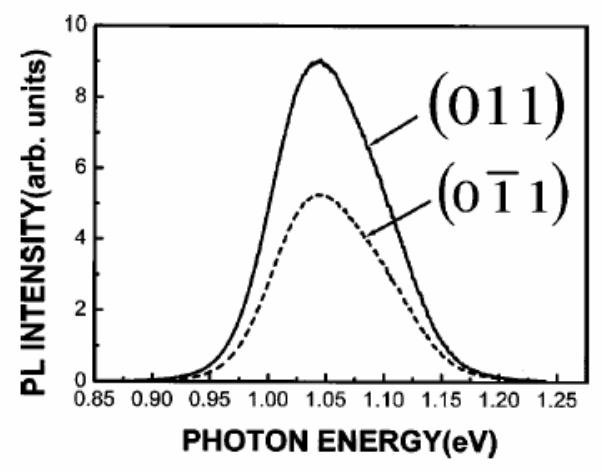

Fig. 1 Polarized photoluminescence spectra of $\mathrm{ZnTe} / \mathrm{CdSe} \mathrm{MQWs}$ under cw excitation

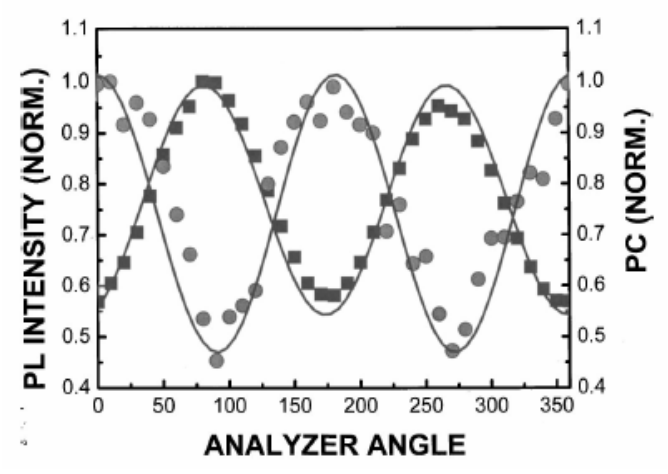

Fig. 2 Photoluminescence intensity (solid square) and polarized photoconductivity signal around $2.5 \mathrm{eV}$ (solid circle) vs analyzer angle for the $\mathrm{ZnTe} / \mathrm{CdSe}$ interfaces without common atom. The solid curves are $\cos ^{2} \theta$ fit.

Figure 3 shows the PC spectra for the incident radiation for different polarization angles. We can also see that the PC spectra display a strong polarization dependence. The main peak at around $2.36 \mathrm{eV}$ corresponds to the spatially direct transition in the ZnTe layer. It weakly depends on the polarization, which is not our main interesting here. We choose the photoresponse signal at around $2.5 \mathrm{eV}$ to analyze the polarization behavior. Using the same definition of the degree of the polarization defined for PL spectra, we find that the value of $\mathrm{P}$ can be as high as $40 \%$. In addition, we can also see that the polarization dependence of the PC spectra covers a wide range of wavelengths as shown in the Figure 4. Figure 3 shows the polarization dependence of the PC response at around $2.5 \mathrm{eV}$. The data can also be described by $\operatorname{the}^{2} \theta$ rule. The maximum and minimum signal correspond to the polarization along $(011)$ and $(0 \overline{11})$, respectively, which is similar to the behavior of the PL spectra. It indicates that the anisotropic PC response arises from the interface chemical bonds. However, the wide range response of the PC spectra as shown in Fig. 3 does not solely come from the type-I and type-II band to band transitions. The transition of the continuous energy states can be attributed to interface imperfections. For example, there may exist wrong bonds at interface (e.g., $\mathrm{Zn}-\mathrm{Se}$ and $\mathrm{Cd}-\mathrm{Te}$ bonds in $\mathrm{ZnTe} / \mathrm{CdSe}$ heterostructures), which are different from the bonds in constituent layers. These wrong bonds can be considered as a kind of localized distortion, i.e., a certain "defectlike" impurity, with respect to the host structure. Close examination of high resolution transmission electron microscope (TEM) spectra shows the existence of reconstruction at interfaces in ZnTe/CdSe [6]. Thus, polarized PC spectra provide a very good opportunity to obtain the effects of microscopic interface defects in semiconductor heterostructures. It is worth noting that PL and PC polarization curves are shifted by $90^{\circ}$. It may be attributed to the fact that the PL signal arises from the electron-hole recombination and the PC response is dominated by the remaining electrons and holes in the extended states. Thus, when the PL intensity is larger, the PC signal will become smaller. 


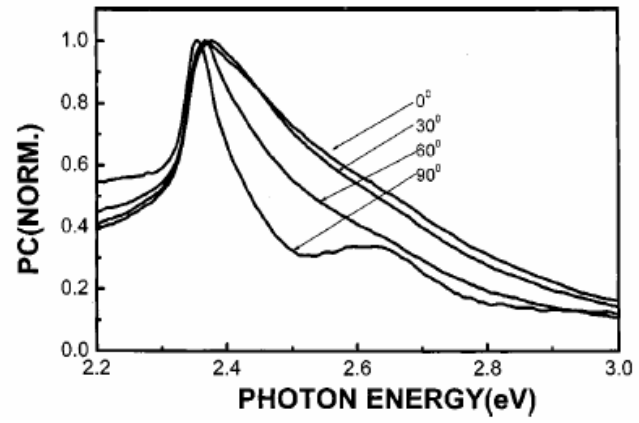

Fig. 3 Polarized photoconductivity spectra of $\mathrm{ZnTe} / \mathrm{CdSe}$ MQWs.

4 Summary In summary, a strong polarization dependence has been observed in type-II CdSe/ZnTe QWs by photoluminescence and photoconductivity measurements. We show that the effects of anisotropy arise from the inherent nature of the interface chemical bonds in type-II heterostructures without a common atom. In addition, we point out that polarized PC measurement is a sensitive tool to probe the microscopic property of interface imperfection.

Acknowledgements This research was supported by the National Science Council and Ministry of Education of the Republic of China. We thank Dr. J. K. Furdyna and Dr. H. Luo for providing the studied samples.

\section{References}

[1] I. F. Nye, in: Physical Properties of Crystal (Oxford University Press, London, 1957).

[2] O. Krebs and P. Voisin, Phys. Rev. Lett. 77, 1829 (1996).

[3] M. S. Yeganeh, J. Qi, A. G. Yodh, and M. C. Tarmargo, Phys. Rev. Lett. 69, 3579 (1992).

[4] N. Samarth, H. Luo, J. K. Furdyna, S. B. Qadri, Y. R. Lee, R. G. Alonso, E. K. Suh, A. K. Ramdas, and N. Otsuka, Surf. Sci. 228, 226 (1990).

[5] N. Samarth, H. Luo, J. K. Furdyna, S. B. Qadri, Y. R. Lee, A. K. Ramdas, and N. Otsuka, Appl. Phys. Lett. 54, 2680 (1989).

[6] H. Luo, N. Samarth, F. C. Zhang, A. Pareek, M. Dobrowolska, J. K. Furdyna, K. Mahalingam, N. Otsuka, W. C. Chou, A. Petrou, and S. B. Qadri, Appl. Phys. Lett. 58, 1783 (1991).

[7] S. M. Tseng. Y. F. Chen, Y. T. Cheng, C. W. Hsu, Huang, and D. Y. Lin, Phys. Rev. B 64, 195311 (2001).

[8] H. J. Chang, Y. F. Chen, H. P. Lin, and C. Y. Mou, Appl. Phys. Lett. 78, 3791 (2001).

[9] E. T. Yu, M. C. Phillips, J. O. McCaldin, and T. C. McGill, J. Vac. Sci. Technol. B 9, 2233 (1991).

[10] M. Schmidt, M. Grun, S. Petillon, E. Kurtz, and C. Klingshirn, Appl. Phys. Lett. 77, 85 (2000).

[11] B. V. Shanabrook and B. R. Bennet, Phys. Rev. B 50, 1695 (1994). 